Musikhören mit dem MP3-Player

\section{Kommt der Schrittmacher aus dem Takt?}

Moderne Mini-Audiogeräte, sogenannte $\mathrm{MP}_{3}$-Player, können die Funktion von Herzschrittmachern stören. So lautete zumindest das beunruhigende Ergebnis einer kleinen Studie aus den USA. MMW fragte den Kardiologen und Rhythmologen Dr. Johannes Sperzel von der Kerckhoff-Klinik in Bad Nauheim, wie sicher Schrittmacherpatienten in unserer von elektromagnetischen Strahlenquellen übervollen Umwelt sind.

MMW: Wie beurteilen Sie die US-Studie, wonach der iPod zu Interferenzen mit Herzschrittmachern führen kann? Sperzel: Herzschrittmacher und implantierbare Defibrillatoren (ICD) sind sehr sichere Geräte. Letztlich handelt es sich bei Wechselwirkungen mit elektromagnetischen Strahlenquellen stets um eine Dosis-Wirkungs-Beziehung. Wenn sich jemand eine Stunde lang direkt an die Diebstahlsicherung eines Geschäfts anlehnt, dann wird das irgendwann auch dem besten Schrittmacher zu dumm. Bei den Messungen in der Studie war der iPod fünf bis zehn Sekunden direkt über der Implantationsstelle des Schrittmachers positioniert worden - eine extrem seltene Situation. Die bei einigen Patienten protokollierten Interferenzen waren nicht bedrohlich.

MMW: Halten Sie die Studie also für praktisch irrelevant?

Sperzel: Ich möchte jeden Kollegen bitten, sich in solchen Gesprächen mit Patienten folgende entscheidende Frage zu stellen: Ist der betreffende Patient vital abhängig vom Herzschrittmacher oder hat das Gerät lediglich unterstützende Funktion? Ist der Patient nicht vital abhängig, und das ist meistens der Fall, braucht er keine Angst vor einem Geräteversagen zu haben. Selbst wenn dieser äußerst seltene Fall eintreten würde, wäre er nicht akut gefährdet. Bei vital vom Gerät abhängigen Menschen wäre die Folge des Stimulationsausfalls eine kurzfristige Synkope. Jedoch würde das System nach Entfernung aus der Stör-

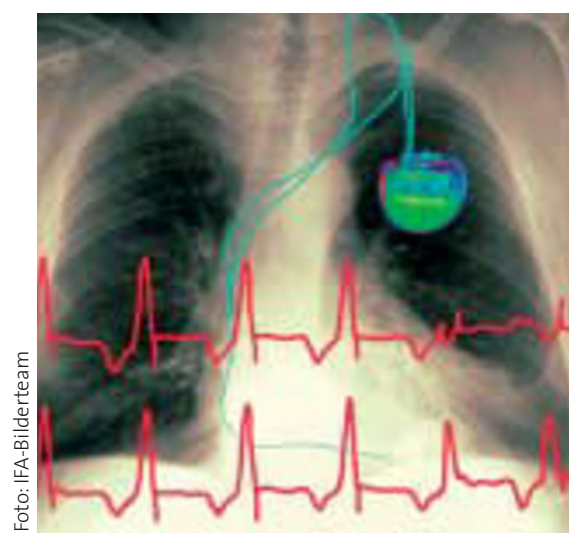

Die meisten Patienten sind nicht vital vom Schrittmacher abhängig. Ein Stimulationsausfall bringt sie nicht in akute Gefahr.

quelle sofort wieder anfangen zu arbeiten. Wer in normaler Geschwindigkeit durch ein Flugsicherungsgerät oder an Diebstahldetektoren entlangläuft, dem kann eigentlich nichts passieren.

MMW: Welche Patienten sind vital abhängig von diesen Geräten? Sperzel: Meist handelt es sich um Patienten mit kompletter Überleitungsblockierung zum AV-Knoten, was z. B. nach einem operativen Aortenklappenersatz oder nach dem Verschluss eines hochsitzenden Ventrikelseptumdefekts der Fall sein kann.

MMW: MP3-Player, Mobiltelefone, schnurlose Festnetztelefone, Sicherheitsschleusen - alles kein Problem?

Sperzel: Wenn jemand sein 15 Jahre altes Handy, das wesentlich mehr elektromag-

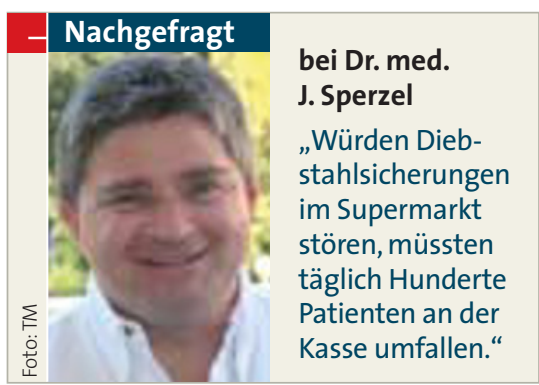

netische Strahlung abgibt als heutige Geräte, unmittelbar über dem Schrittmacher trägt und es handelt sich um ein unipolares Elektrodensystem, dann kann es vielleicht zu Interferenzen kommen. Aber selbst dann enden sie im schlimmsten Fall in einer starr frequenten Stimulation. Das Durchgehen durch die elektronische sicherheitsschleuse am Flughafen sollte man den Patienten aus psychologischen Gründen ersparen, weil sie das Titangehäuse des Schrittmachers detektiert. Gefährlich ist das nicht, das kann ich zumindest für europäische Flughäfen sagen. Jeder Supermarkt hat heute versteckte Sicherheitssysteme installiert. Würden sie tatsächlich die Schrittmacher stören, müssten wir täglich Hunderte Patienten sehen, die beim Schlangestehen an der Kasse umgefallen sind.

Kabellose Telefone sind kein Problem, Hochspannungsleitungen sind kein Problem, Mikrowellen sind kein Problem! Der Zahnarzt kann seine Ultraschallbehandlung zur Zahnsteinentfernung vornehmen. Der Chirurg darf den Elektrokauter benutzen, wenn er sich vorher über den $\mathrm{Pa}$ tienten kundig macht und den bipolaren Kauter benutzt.

MMW: Wie viele Zwischenfälle mit Schrittmachersystemen haben Sie erlebt? Sperzel: In 15 Jahren, in denen ich eine fünfstellige Anzahl von Kontrollen vorgenommen habe, habe ich zwei Systeme mit Komplettausfall erlebt: einmal nach einer kernspintomografischen Untersuchung, die mit modernen Geräten heute durchaus möglich ist, und einmal nach mechanischer Gewalteinwirkung.

- Interview: Dr. Thomas Meißner

Haben Sie noch Fragen an Dr. Sperzel? Sie erreichen ihn über die MMW-Redaktion: Fax: 089-4372-1399

E-Mail: schumacher@urban-vogel.de 